\title{
EXTENDED LYMPHADENECTOMY IN BLADDER CANCER DURING RADICAL CYSTECTOMY: BENEFIT AND MORBIDITY
}

\author{
Islam Mohiedden M, Diab El-Sayed M, Reda Mohamed S, Saad A M Al Qady, \\ Department of Urology, Zagazig University, Zagazig, Egypt
}

\begin{abstract}
Background: Controversy still presents regarding the ideal proximal extension of lymphadenectomy at radical cystectomy.

Objective: To provide an accurate map of lymph node metastasis in patients with bladder cancer and to evaluate benefit and morbidity of extended lymphadenectomy.

Patient and method: A Prospective study of 65 patients with bladder transitional cell carcinoma with stage T2-T4a-Nx-M0 undergoing radical cystectomy with mapping extended lymphadenectomy was completed between September 2011 and March 2015 at the Department of Urology, Zagazig University Hospitals.

Intervention: Radical cystectomy with mapping extended lymphadenectomy.

Results: The mean \pm SD total of retrieved lymph node number in the study population was 28.7 \pm 9.8 . Twenty of 65 patients (30.7\%) had positive LN. LN metastases above bifurcation of common iliac artery are common (40\% of patients with positive lymph node had metastasis in common iliac lymph nodes and presacral lymph nodes). No skip metastasis to above common iliac bifurcation LN. Negative LN patients had better survival than positive LN patients and survival did not affected by location, number and density of positive $\mathrm{LN}$.

Conclusions: Extended lymphadenectomy till level of bifurcation of aorta provide better identification of positive lymph node correctly and good assign of pathological node metastasis stage in node positive cases without significant morbidity.
\end{abstract}

Keywords: Extended lymphadenectomy, radical cystectomy, bladder cancer, lymph node, , lymph node density.

\section{INTRODUCTION}

Padical cystectomy combined with - Lymphadenectomy is considered the standard management for invasive bladder cancer patients. About $25 \%$ of patients have metastases at lymph node during radical cystectomy, and the role of lymphadenectomy in staging is unequivocal (1). Leadbetter in 1950 , (2) described a technique for lymphadenectomy that is used still now.

Surgical treatment of invasive bladder cancer by radical cystectomy and lymphadenectomy evolved more than Seventy years ago ${ }^{(3)}$. At 1950, Kerr noticed that the local recurrence rate after cystectomy decreased significantly when combined with lymphadenectomy pelvic lymph nodes (4). Whitmore and Marshall (5) registered that 5year survival after radical cystectomy and pelvic lymphadenectomy was $16 \%$ for patients with positive nodes. In 1982, Skinner made interest in this subject when he published paper with title "Management of invasive bladder cancer: a meticulous pelvic node dissection can make a difference." (6)

However, the proximal level of lymphadenectomy or the minimal lymph nodes number that should be removed has not been determined, and its role in prognosis is still under controversy. ${ }^{(7,8)}$

Recent investigations have noticed that extension of lymphadenectomy to include presacral and common iliac LNs improved survival ${ }^{(9)}$. This is depend on lymph nodes mapping studies showing that lymph nodes metastasis above the common iliac artery bifurcation occurs with significant percentage (2, 10-13), and multiple papers noticed that increasing number of removed lymph nodes improved survival ${ }^{(\mathbf{9 , 1 4 , 1 5})}$.

In this study, we evaluate the benefit and morbidity of extended lymphadenectomy to level of inferior mesenteric artery in bladder cancer patients at radical cystectomy.

\section{PATIENTS AND METHODS}

This prospective study was carried out at the Department of Urology, Faculty of Medicine, Zagazig University Hospitals between September 2011 and March 2015.

Informed consent was obtained from every patient. Approvals was obtained from the ethical committee in faculty of medicine Zagazig University and from patients included in the study. 
The study included 65 patients (51men (78\%) and 14 women (22\%) diagnosed as TCC (T2-T4a, N0-Nx, M0). Patients were treated by radical cystectomy and extended lymphadenectomy till level of inferior mesenteric artery.

Patients with histopathology of SCC and Adenocarcinoma, previous Pelvic radiotherapy, previous neoadjuvant chemotherapy for bladder cancer, previous pelvic lymphadenectomy and patient with bad general condition (performance status $>2$ according to WHO classification ${ }^{(16)}$ ) were excluded from this study.

Operation: The procedure was performed by only one team. Extended lymphadenectomy was done for all patients till the level of origin of the inferior mesenteric artery, the anatomical borders were at the genitofemoral nerve laterally and the bladder wall medially. LN dissection was started at the level of inguinal ligament and extended proximally. The external iliac nodes, defined as tissues between the external iliac artery and the psoas minor muscle, were removed. Internal iliac nodes (located in tissues between the obturator nerve and bladder wall) were dissected and removed, including the tissue on the anterior surface of the anterior division of the internal iliac artery. Obturator group of nodes, defined as tissues between the external iliac vein laterally and the obturator nerve posteromedially was exposed and removed by skeletonization and retraction of external iliac vien laterally. Dissection was then carried distal to aorta and inferior vena cava to remove the lymphatic tissues along the common iliac arteries (common iliac nodes) and in the intercommon iliac region (presacral nodes). Para aortic lymph nodes defined as fibrolymphatic tissues lateral to and in front of aorta were removed till level of inferior mesenteric artery origin. Paracaval lymph nodes defined as fibrolymphatic tissues lateral to and in front of IVC and between the aorta and IVC were removed till level of inferior mesenteric artery origin. LNs were submitted in 11 separate nodal packets: Lymph node packets: (1) Paraaortic LN (2)Paracaval LN (3)Presacral $(4,5)$ right (Rt) and left (Lt) common iliac, , $(6,7) \mathrm{Rt}$ and Lt external iliac, $(8,9)(\mathrm{Rt})$ and $(\mathrm{Lt})$ obturator $(10,11) . \mathrm{Rt}$ and Lt internal iliac. Figure 1

For analytic purposes, the LND regions were divided into three anatomic levels (Figure 1). Level I: Standard lymphadenectomy (Below common iliac bifurication). Level II: Extended lymphadenectomy (Regions between bifurcations of common iliac vessels and aortic bifurcation). Level III: Super extended lymphadenectomy (Regions between bifurcations of aorta till the inferior mesenteric artery (IMA)).

Bipolar diathermy was used for hemostasis. Lymphadenectomy operation time was recorded from time of starting LN dissection till the end of dissection of lymphadenectomy at each level. Blood loss during each level was measured by amount of blood in suction and weighting of towel.

\section{Pathological}

evaluation:

Lymphadenectomy specimens are placed as quickly as possible in fixative $(10 \%$ buffered formalin) so that the tissue does not degrade before pathologic evaluation and each anatomical group was put in separate container. Tumor histopathological type, stage, grade, size and site were determined. The number of retrieved nodes per anatomical group was determined. Number of positive nodes in each anatomical group was determined. Lymph nodes density was determined [Total no of positive LN /total no of retrieved $\mathrm{LN}$ ].

\section{FOLLOW-UP:}

\section{Short term follow up:}

Early postoperative morbidity was defined as that within 30 days after surgery. Patients were examined daily searching for complication of lymphadenectomy (lymphocele, D.V.T, pulmonary embolism). Pelvic ultrasound and Doppler lower extremity studies were performed in all patients with signs and/or symptoms of lymphocele. All pelvic lymphocele, DVTs, and PEs were confirmed radiographically.

\section{Long term follow up:}

Follow-up was performed according to the following protocol. In general, patients were seen at 3 and 6 months after surgery then 
every 6 months for 2 years. Follow-up visits including the following: Physical examination, serum chemistry evaluation, CT abdomen and chest radiography were performed at least annually or when clinically indicated and chest $\mathrm{CT}$ and bone scans were performed if clinically indicated.

Disease recurrence was defined as any radiographic or pathologically documentation of disease recurrence either local or systemic. Time to recurrence was calculated as the time from cystectomy to the date of the first documented clinical recurrence. Cancer specific survival was calculated from the time of cystectomy until date of death from bladder cancer. The cause of death was determined by using information collected from the treating physicians, family interrogation, chart review, or death certificates alone. Cause of death was prospectively entered and verified by chart review.

\section{STATISTICAL ANALYSIS}

According to the type of data, the following tests were used to test differences for significance; Differences between frequencies (qualitative variables) and percentages in groups were compared by Chisquare test $\left(\mathrm{X}^{2}\right)$. Differences between means (quantitative variables) IN multiple parametric groups by ANOVA (F) test. Kappa agreement was used to test the agreement. $\mathrm{P}$ value was set at $<0.05$ for significant results $\&<0.001$ for high significant result.

Recurrence free and overall survival rates were calculated according to KaplanMeier actuarial method from the time of diagnosis. The statistical package used was XLSTAT Version 2015.1.01.

\section{Patient characteristics:}

\section{RESULTS}

This study included 65 patients (51men (78\%) and 14 women (22\%). the mean age was $56 \pm 4.4$ years. Mean BMI was 30.9 \pm 4.5 $\mathrm{kg} / \mathrm{m} 2$.

\section{Tumor characteristics:}

table 1.

Tumor character was summarized in

\section{Distribution of Lymph node metastases:}

Of 65 patients, the mean \pm SD of retrieved LNs were $28.7 \pm 9.8$. Tumor positive lymph nodes were found in 20 of 65 patients
(30.7\%). Tumor positive lymph nodes were found in 20 of 65 patients $(30.7 \%)$. All patients with LN metastases in the common iliac or aortic bifurcation region (level II and level III) had metastases at level I dissection (obturator, external iliac and internal iliac L.N. groups) (no skipped area). Eight patients $(12.3 \%)$ have LNs metastasis above common iliac bifurcation and 1 patient (1.5\%) has LNs metastasis above aortic bifurcation.

\section{(Table 2)}

The percentage of patients with nodal metastases increased significantly with the pT category of the primary bladder tumor (Table 3). Eight cases from 34 patients $(23.5 \%$ of this group) with pT2 showed +ve LNs, six cases from 17 patients with pT3 showed +ve LNs (35\% of this group) and finally six cases from 14 patients with pT4 showed +ve LNs (42.8\% of this group) according to TNM staging system ${ }^{(17)}$.

Number and means SD of retrieved LNs in patients with positive LNs and patients with negative LNs were presented in table 5.

Patients with higher stages pT3 or pT4 frequently had LN metastases outside of the common boundaries for standard LN dissection. Patients with stage (pT2) had two patients with LNs metastasis above common iliac bifurcation (5.8\% of their group); stage pT3 have three patients with LNs metastasis above common iliac bifurcation $(17.6 \%$ of their group); stage pT4a had three patients (21\% of their group) with LNs metastasis above common iliac bifurcation. (Table 6)

In our study we had 20 patients with positive $\mathrm{LN}$, we calculated the $\mathrm{LN}$ density for those patients [Total no of positive LN /total no of retrieved $\mathrm{LN}]$ and we found that the mean LN density for these patients $14.22 \% \pm$ $11.3 \%$ SD. 13 patients (60\%) had LN density less than $20 \%$. Seven patients had LN node density more than $20 \%$. (Table 7|)

In this study, we had 8 cases $(12.3 \%$ of all patients) (40\% of patients with $\mathrm{LNs}$ metastasis) which have only a single lymph node metastasis. In these 8 patients, single lymph node was positive and all positive lymph nodes are located in Level I (obturator, external iliac and internal iliac L.N. groups).

\section{Survival and recurrence:}


The follow-up period present in this study ranged from 6 to 42 months with a median of 26 months. During follow-up, 19 patients $(29.2 \%)$ died, $6(9.2 \%)$ of them died from comorbid disease and 13 of them $(20 \%)$ died from bladder cancer recurrence. Eighteen patients $(27.6 \%)$ were diagnosed with a recurrence. The cancer specific survival and recurrence-free survival rates at 2 years in the entire study population were $75 \%$ and $65 \%$, respectively. (Figure 2)

Stratifying patients according to lymph node positivity, there was significant difference between node positive patients and node negative patients in the CSS and RFS at 2 years $(\mathrm{p}=0.006$ and $\mathrm{p}=0.027$, respectively). (Figure 3)

Stratified by +ve LN level, there was insignificant difference between Patients with positive LNs above Common iliac bifurcation and patients with positive LNs confined to below common iliac bifurcation regions in the CSS and RFS at 2 years $(\mathrm{p}=0.795$ and $\mathrm{p}=$ 0.427 , respectively). (Figure 4)

Classifying +ve LN patients according to LN density percentage, no significant difference was seen between patients with LN density $<20 \%$ and patients with LN density $>20 \%$ in CSS and RFS ( $\mathrm{p}=0.978$ and $\mathrm{p}=$ 0.539 , respectively). (Figure 5)
Classifying +ve LN patients according to number of positive $\mathrm{LN}$, no significant difference was seen between patients with one positive $\mathrm{LN}$ and patients with more than one positive $\mathrm{LN}$ in CSS and RFS at 2 years $(p=0.344)(p=0.406)$ respectively. (Figure 6)

Perioperative morbidity of extended lymphadenectomy:

- No cases of nerve injury (obturator, genitofemoral, femoral nerves) were reported.

- Operation time was reported in table 8.

- Perioperative blood loss was reported in table 8 .

- Four cases (6\%) of lymphocele formation postoperative were detected by follow up ultrasound with diagnostic aspiration and chemical analysis. All of them were asymptomatic and managed conservatively except one case $(1.5 \%)$. This case complained from abdominal distention, abdominal pain and bilateral lower limb edema. This case was managed by percutaneous drainage ultrasound guiding. (Table 9)

- Only one case (1.5\%) of DVT was seen. Only one case $\mathbf{( 1 . 5 \% )}$ of pulmonary embolism was seen. Lymphoedema was not seen in any case.

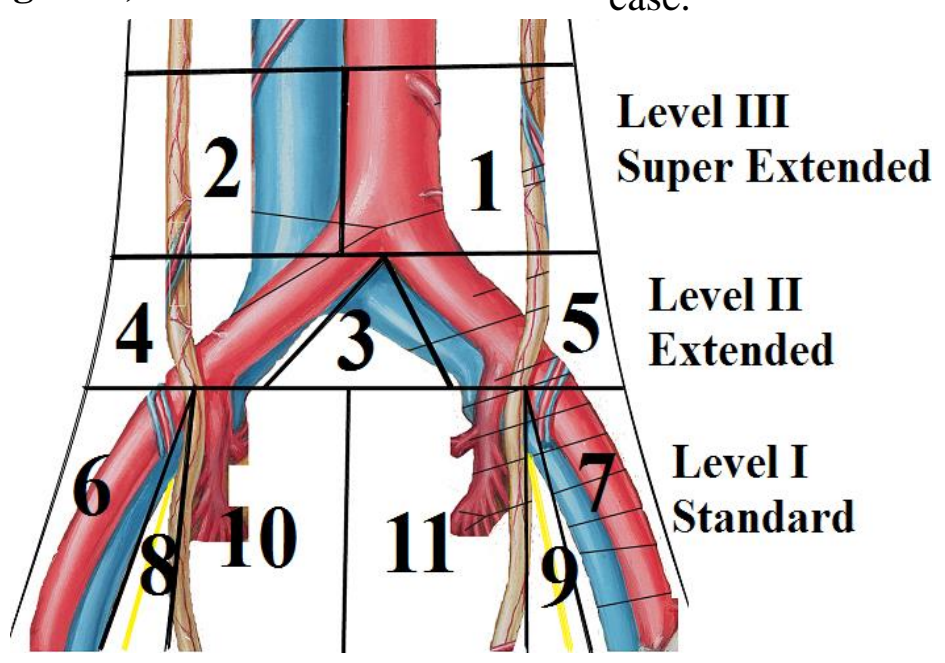

Figure 1: The boundaries of a standard (I), Extended (II), and Super extended (III) PLND. Lymph node packets: (1) Paraaortic LN (2)Paracaval LN (3)Presacral $(4,5)$ right (R) and left (L) common iliac, , $(\mathbf{6}, 7) \mathrm{R}$ and $\mathrm{L}$ external iliac, $(\mathbf{8}, \mathbf{9})(\mathrm{R})$ and $(\mathrm{L})$ obturator $(\mathbf{1 0}, \mathbf{1 1})$. R and $\mathrm{L}$ internal iliac. 
Table1: Tumor characteristics

\begin{tabular}{|c|c|}
\hline Variable & No (\%) \\
\hline 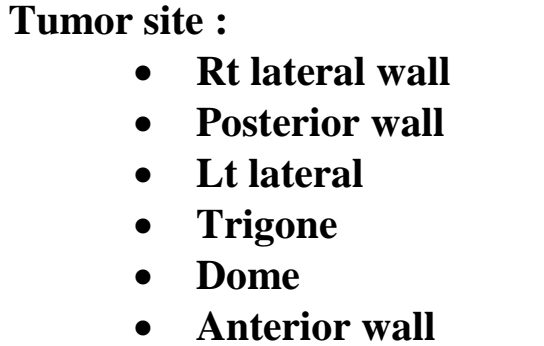 & $\begin{array}{ll}21 & (32.3) \\
17 & (26) \\
12 & (18.4) \\
8 & (12.3) \\
6 & (9.2) \\
1 & (1.5)\end{array}$ \\
\hline $\begin{array}{r}\text { Tumor size }: \\
\bullet \quad<5 \mathrm{~cm} \\
\bullet \quad \geq 5 \mathrm{~cm}\end{array}$ & $\begin{array}{ll}28 & (43) \\
37 & (57)\end{array}$ \\
\hline $\begin{aligned} \text { Tumor grade : } \\
\bullet \quad \text { Grade II } \\
\bullet \quad \text { Grade III } \\
\end{aligned}$ & $\begin{array}{ll}25 & (38.4) \\
40 & (61.6)\end{array}$ \\
\hline $\begin{aligned} \text { Stage : } & \\
\bullet & \text { T2 } \\
\bullet & \text { T3 } \\
\bullet & \text { T4a }\end{aligned}$ & $\begin{array}{ll}34 & (52.3) \\
17 & (26) \\
14 & (21.5)\end{array}$ \\
\hline
\end{tabular}

Table 2: Percentage of LNs metastasis patients in each level

\begin{tabular}{|c|c|c|c|c|c|c|}
\hline & & $+\mathrm{VE}$ & $-\mathrm{VE}$ & $\begin{array}{l}\text { Total patients with } \\
\text { +ve LNs }\end{array}$ & $\mathbf{X}^{2}$ & $\overline{P \text { value }}$ \\
\hline +ve Level 1 & No (\%) & $20(100 \%)$ & $\mathbf{0}$ & $20(100.0 \%)$ & \multirow{3}{*}{44.6} & \multirow{3}{*}{$<0.001$} \\
\hline +ve Level 2 & No (\%) & $8(40 \%)$ & $12(60 \%)$ & $20(100.0 \%)$ & & \\
\hline +ve Level 3 & No $(\%)$ & $1(5 \%)$ & $19(95 \%)$ & $20(100.0 \%)$ & & \\
\hline
\end{tabular}

Table 3: Patients with lymph node metastases ( $\left.p N_{2}\right)$ in each pT category

\begin{tabular}{lllllll}
\hline pT Category & T2 & T3 & T4a & total & P \\
\hline+ VE & $\mathbf{8}(23.5 \%)$ & $6(35 \%)$ & $6(42.8 \%)$ & $20(30.7 \%)$ & \\
\hline -VE & $26(76.5 \%)$ & $11(65 \%)$ & $\mathbf{8}(57.2 \%)$ & $45(69.3 \%)$ & $<0.001$ \\
\hline Total & $34(100 \%)$ & $17(100 \%)$ & $14(100 \%)$ & $65(100 \%)$ & \\
\hline
\end{tabular}

Table 4: Patients with lymph node metastases in each pT categoryin each level

\begin{tabular}{|c|c|c|c|c|c|}
\hline pT Category & & $\mathrm{T} 2$ & T3 & T4a & total \\
\hline Total No. Pts & & $34(52.3 \%)$ & $17(26 \%)$ & $14(21.5 \%)$ & $65(100 \%)$ \\
\hline +ve Level 1 & No (\%) & $8(23.5 \%)$ & $6(35 \%)$ & $6(42.8 \%)$ & $20(30.7 \%)$ \\
\hline +ve Level 2 & No (\%) & $2(5.8 \%)$ & $3(17.6 \%)$ & $3(21 \%)$ & $8(12.3 \%)$ \\
\hline +ve Level 3 & No $(\%)$ & $\mathbf{0}$ & $\overline{0}$ & $1(7 \%)$ & $1(1.5 \%)$ \\
\hline Total Pts +ve (\%) & & $8(23.5 \%)$ & $6(35 \%)$ & $6(42.8 \%)$ & $20(30.7 \%)$ \\
\hline
\end{tabular}


Table 5: Number and mean SD of retrieved LNs in patients with +ve and -ve LNs.

\begin{tabular}{llllll}
\hline & $\begin{array}{l}\text { No of cases } \\
(\%)\end{array}$ & $\begin{array}{l}\text { No } \\
\text { retrieved LN }\end{array}$ & $\begin{array}{l}\text { Mean } \pm \text { SD of P value } \\
\text { retrieved LN }\end{array}$ & 0.002 \\
\hline Pts with positive LNs & $\begin{array}{l}20 \\
(30.7 \%)\end{array}$ & 661 & $33.05 \pm 9.7$ & \\
\hline Pts with negative LNs & $\begin{array}{l}45 \\
(69.3 \%)\end{array}$ & 1205 & $26.7 \pm 7.4$ & \\
\hline Total & 65 & 1866 & $28.7 \pm 8.6$ & \\
\hline
\end{tabular}

Table 6: Number and percentage of patients with positive LNs above level $I$ in each pT stage according to TNM staging system.

\begin{tabular}{llll}
\hline pT Category & No of total pts & $\begin{array}{l}\text { No of pts with +ve LN above } \\
\text { Level 1 (\%) }\end{array}$ & P value \\
\hline T2 & 34 & $2(5.8 \%)$ & 0.02 \\
\hline T3 & 17 & $3(17.6 \%)$ & \\
\hline T4a & 14 & $3(21 \%)$ & \\
\hline total & 65 & $8(12.3 \%)$ & \\
\hline
\end{tabular}

Table 7:N1 Metastasis in a single lymph node in the true pelvis; N2 Metastasis in multiple lymph nodes in the true pelvis; $\mathbf{N 3}$ Metastasis in common iliac lymph node(s)

\begin{tabular}{|c|c|c|c|c|c|}
\hline \multirow[t]{2}{*}{ L.N density \% } & \multicolumn{3}{|c|}{ pN Status } & \multirow[t]{2}{*}{ Total } & \multirow[t]{2}{*}{ P value } \\
\hline & $\mathbf{N} 1$ & N2 & N3 & & \\
\hline$<20$ & 8 & 2 & 3 & $13(65 \%)$ & \multirow{3}{*}{$<0.001$} \\
\hline$>20$ & $\mathbf{0}$ & 2 & 5 & $7(35 \%)$ & \\
\hline total & 8 & 4 & 8 & 20 & \\
\hline
\end{tabular}

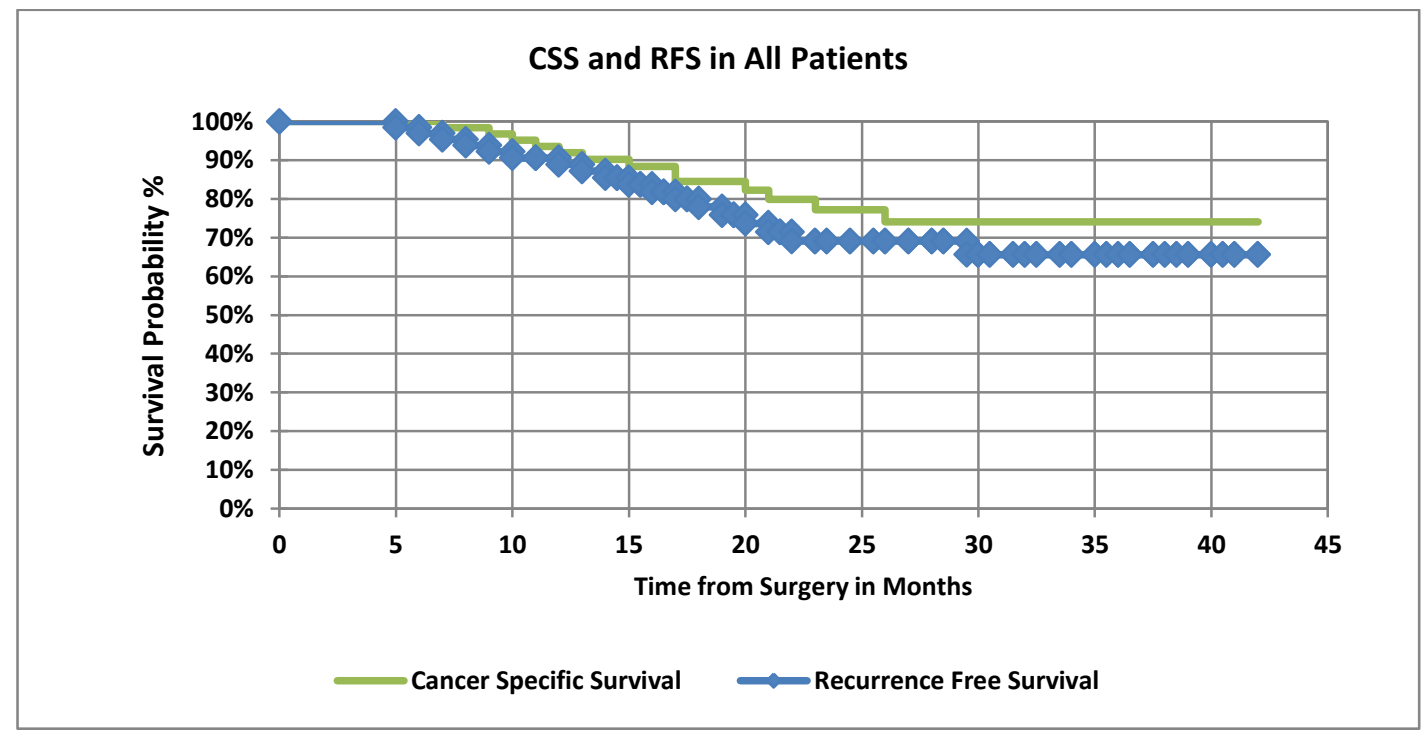

Figure 2: Kaplan-Meier curve for recurrence-free survival and cancer specific survival for all patients. 

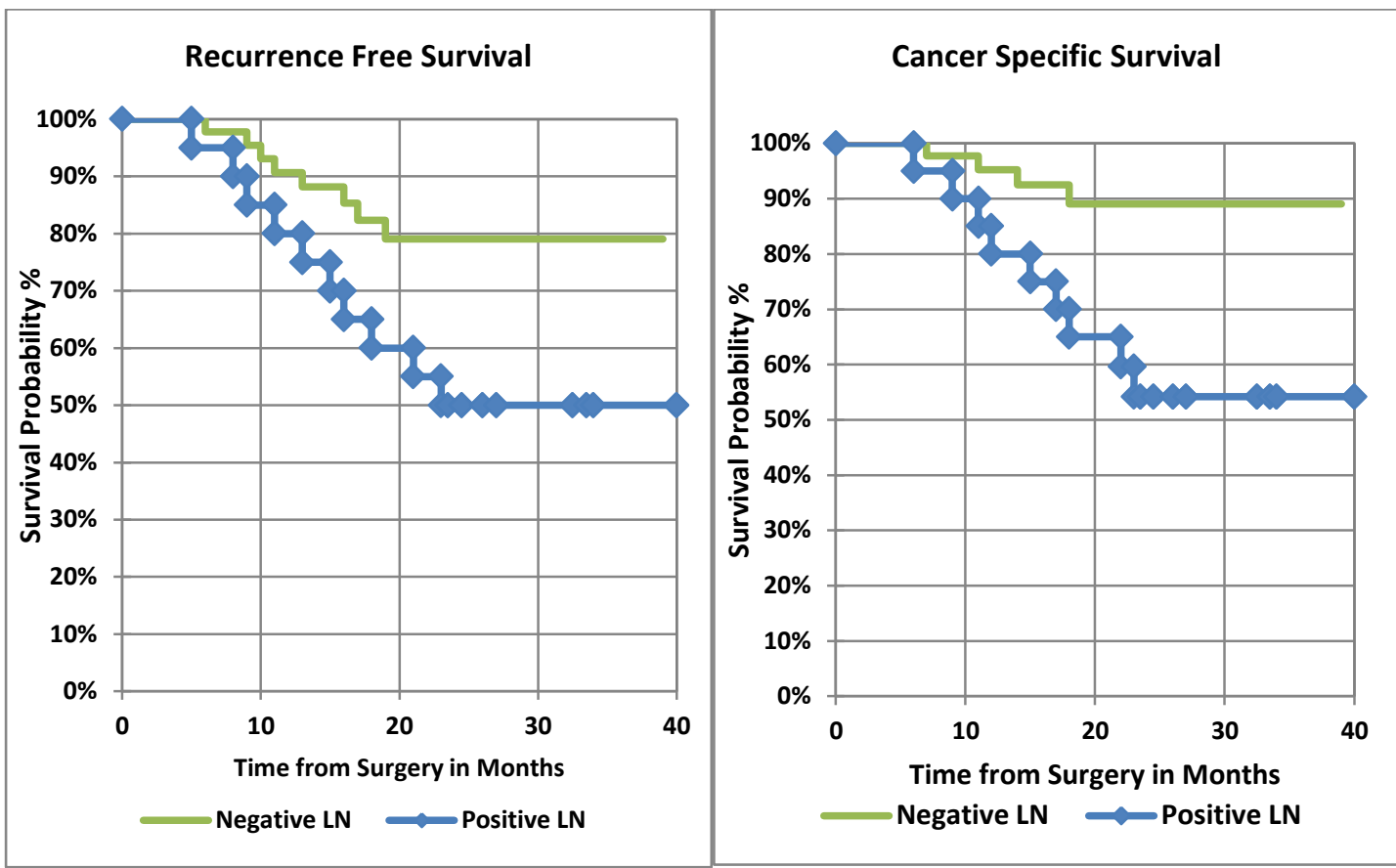

Figure 3: Kaplan-Meier curve for recurrence-free survival and cancer specific survival, stratified by the absence of lymph node involvement ( $\mathrm{pN} 0$ ) or the presence of lymph node involvement $(\mathrm{pN}+)$ at radical cystectomy.
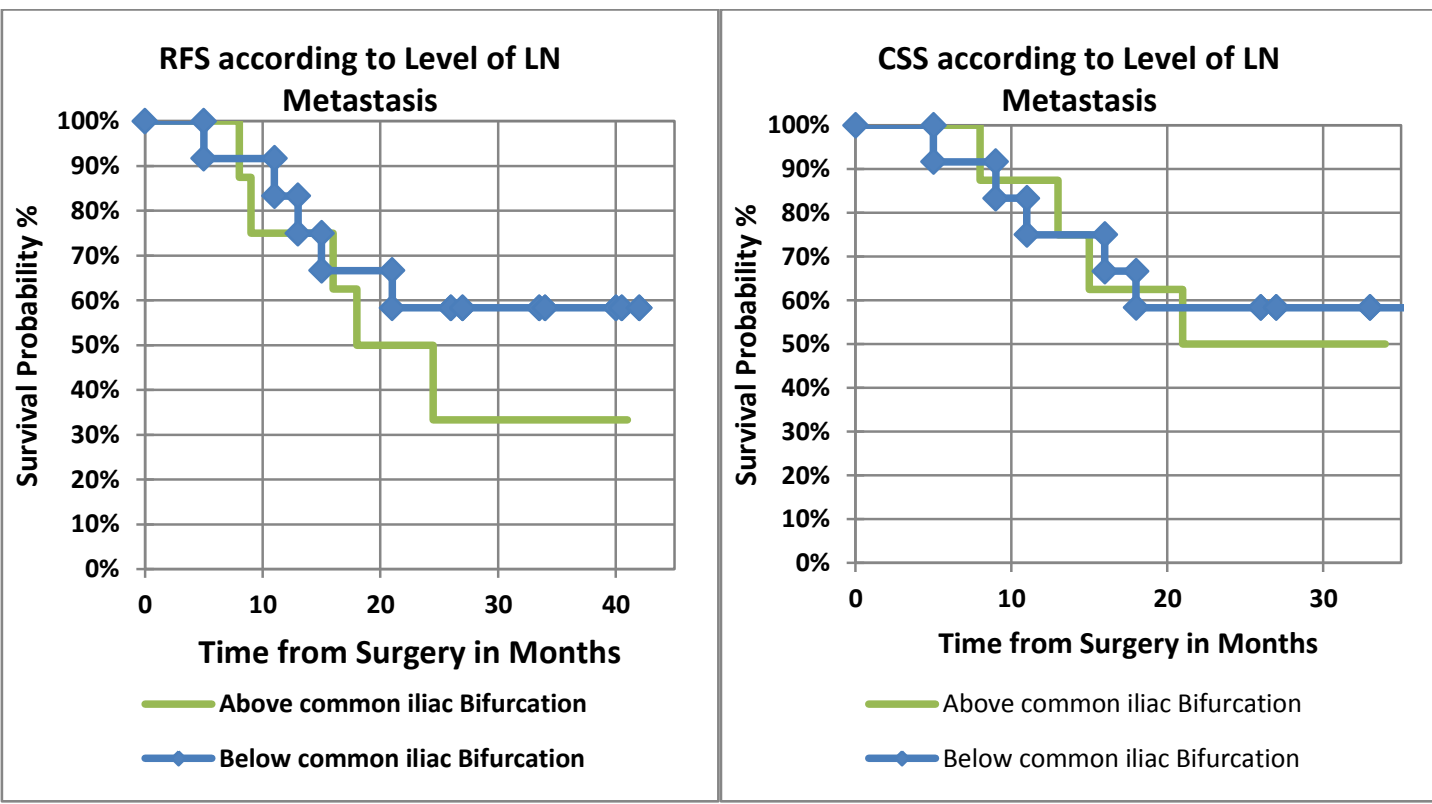

Figure 7: Kaplan-Meier curve for recurrence-free survival and cancer specific survival, stratified by level of positive $\mathrm{LN}$ at radical cystectomy. 

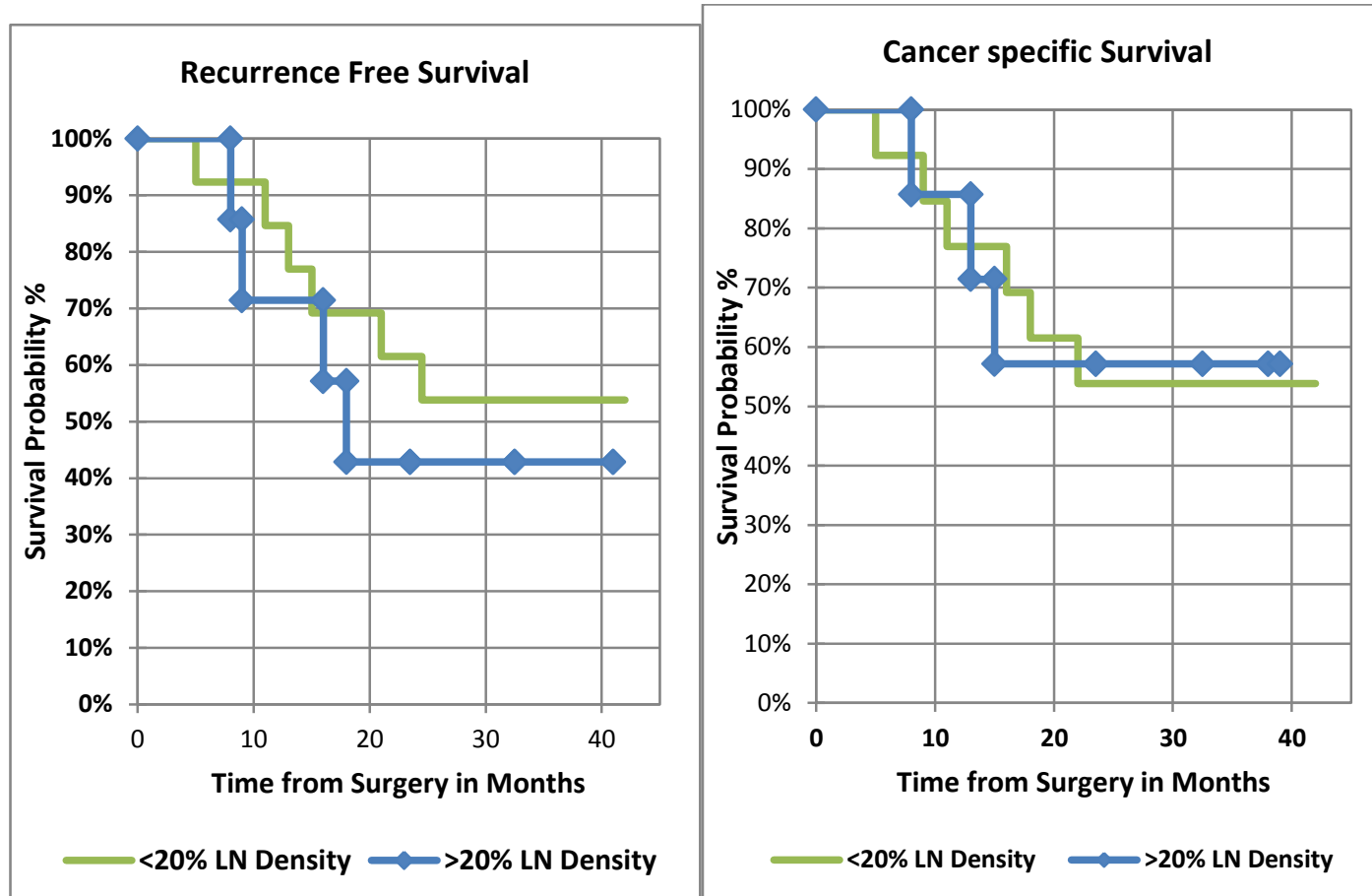

Figure 5: Kaplan-Meier curve for recurrence-free survival and cancer specific survival, stratified by $\mathrm{LN}$ density category $(<20 \% \&>20 \%)$ at radical cystectomy.

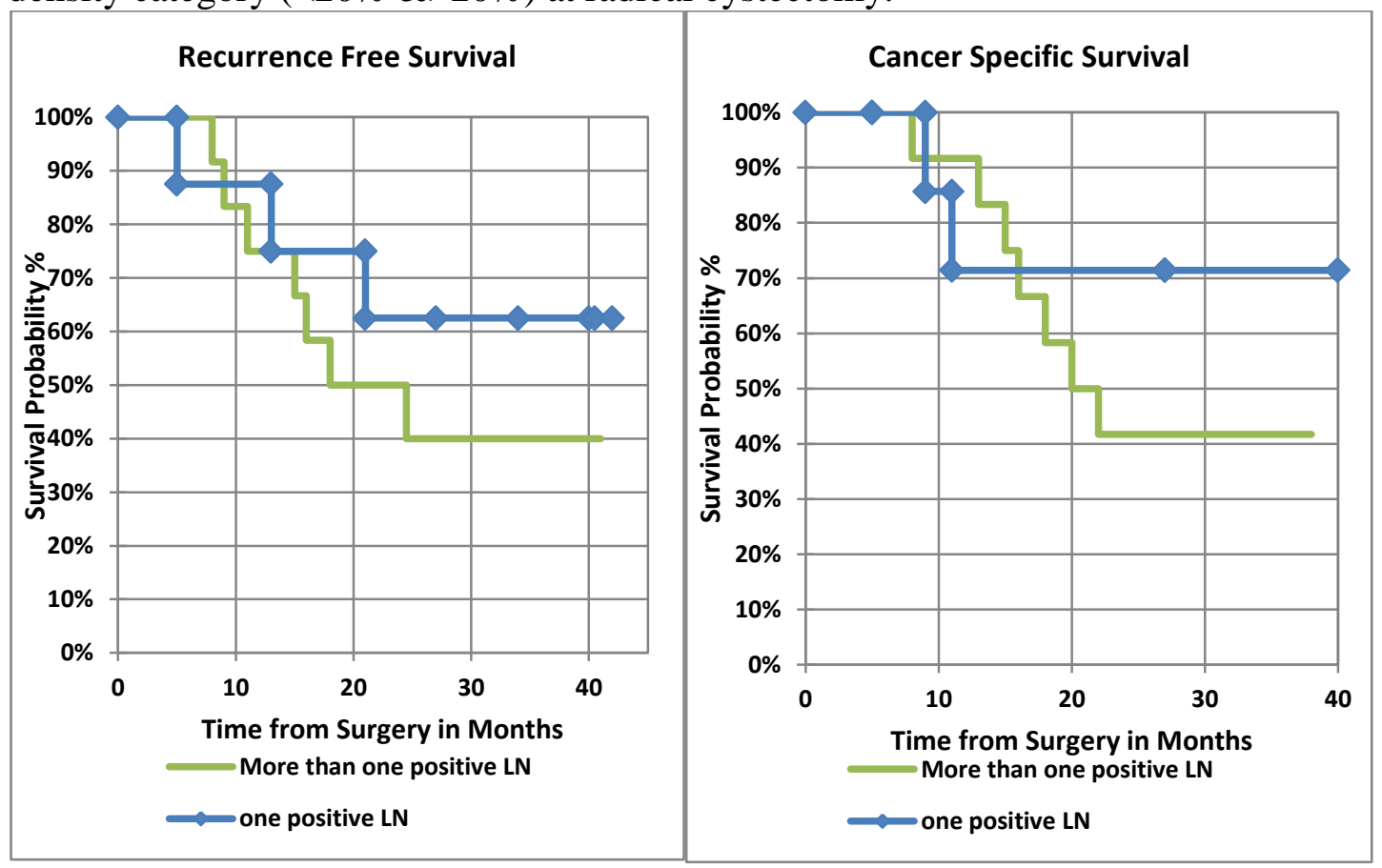

Figure 9: Kaplan-Meier curve for recurrence-free survival and cancer specific survival, stratified by number of positive LN at radical cystectomy..

Table 9: Operating blood loss and time

\begin{tabular}{ccc}
\hline Variable & Mean \pm SD & Range \\
\hline Operative time $($ min) & $324 \pm 54.6$ & $(\mathbf{2 0 5 - 4 3 0 )}$ \\
\hline Operative blood loss $($ cc) & $\mathbf{8 5 0} \pm 180.4$ & $(\mathbf{2 5 0}-4000)$ \\
\hline
\end{tabular}


Table 10: Perioperative complication related to lymphadenectomy

\begin{tabular}{ll}
\hline Complication & No (\%) \\
\hline Lymphocele & 4 cases (6\%) \\
\hline Deep venous thrombosis & $1(\mathbf{1 . 5 \% )}$ \\
\hline Pulmunary embolism & $1(\mathbf{1 . 5 \% )}$ \\
\hline Lymphoedema & 0 \\
\hline Nerve injury & 0 \\
\hline Overall complications & $6(\mathbf{9 . 2 \% )}$ \\
\hline
\end{tabular}

\section{DISCUSSION}

Radical cystectomy combined with lymphadenectomy for invasive bladder cancer patients provides correct staging and better local control ${ }^{(\mathbf{1 8})}$. Lymph node status is one of the strongest prognostic factors in bladder cancer patients ${ }^{(\mathbf{1 8})}$.

Still controversy exists regarding the ideal proximal level of lymphadenectomy, the retrieved lymph nodes number, or the prognostic value ${ }^{(\mathbf{1 1})}$.

No clear recommendation from EAU and AUA guidelines present about the extent of lymphadenectomy ${ }^{\left({ }^{(19)} \text {. The International }\right.}$ Consultation on Urological Diseases guidelines 2012 recommend that common iliac, internal iliac, obturator and external iliac lymph nodes should be removed completely at radical cystectomy ${ }^{(20)}$.

The concept that positive nodes above common iliac artery bifurcation state advanced disease and would not be treated by surgery has made many urologists to undervalue the importance of extended lymphadenectomy.

Our study included 65 patients with 20 patients [30.7\%] had positive LNs this is similar to the results obtained by Dangle and colleagues ${ }^{(22)}$ study include 120 patients with 36 patients $(30 \%)$ had lymph node metastasis, Leissner and colleagues (11) study which included 290 patients with 81 patients [27.9\%] had positive LNs, Abol-Enein and colleagues ${ }^{(23)}$ study included 200 patients and 48 patiens [24\%] showed positive LNs, Dorin and colleagues ${ }^{(24)}$ study include 646 patients with 151 patients [23.4\%] had positive LNs and finally Vazina and colleagues ${ }^{(12)}$ study include 176 patients with 43 patients [24.4\%] had positive LNs.

On the other hand Tarin and colleagues (21) study include 591 patients with 114 patients [19\%] had positive LNs; this difference may be attributed to large number of their patients.

Mean \pm SD of total lymph node retrieved in our study is $28.7 \pm 8.6$. In Vazina and colleagues ${ }^{(12)}$ study, the median number of LNs removed was 25. Dangle and colleagues ${ }^{(22)}$ found that the mean \pm SD of total lymph node retrieved was $36.9 \pm 14.8$.

While Abol-Enein and colleagues (23) found that the mean number of retrieved nodes per patient was $50.6 \pm 14.4$. In Leissner and colleagues ${ }^{(11)}$ study, mean total number and standard deviation of lymph nodes removed was $43.1 \pm 16.1$. The difference in the mean number of nodes retrieved may be due to the difference in number of patients.

We found that percentage of patients with nodal metastases increased significantly with the pT category of the primary bladder tumor. As 8 cases from 34 patients $(23.5 \%$ of this group) with pT2 showed +ve LNs, six cases from 17 patients with pT3 showed +ve LNs (35\% of this group) and finally six cases from 14 patients with pT4 showed +ve LNs (42.8\% of this group)..

These data correlate with Abol-Enein and colleagues ${ }^{(23)}$ results as they showed 48 patients $(24 \%)$ had positive nodes, including 8 of 79 (10\% of patients with pT2 disease) and 40 of $103(38.8 \%$ of patients with _pT3 disease) $(\mathrm{p}=0.001)$.

Also, these data correlate with Vazina and colleagues ${ }^{(\mathbf{1 2})}$ results as no patient with pTis had LN metastases. LN metastases were found in 1 of 28 patients $(3.6 \%)$ with pT1, 10 of 64 patients $(15.6 \%)$ with pT2, 20 of 50 patients $(40 \%)$ with pT3, and 12 of 24 patients $(50 \%)$ with pT4.

Leissner and colleagues ${ }^{(11)}$ showed similar results in his study as they found $\mathrm{LN}$ metastasis in 1 of 57 patients $(1.8 \%)$ with 
pT1, 17 of 93 (18\%) patients with pT2, 53 of 120 (44\%) patients with pT3, and 10 of 20 patients $(50 \%)$ with pT4.

Dorin and colleagues ${ }^{(24)}$ showed LN metastasis in 12 of 243 patients (5\%) with <pT2, 34 of 167 patients (20\%) with pT2, 67 of 174 patients $(38.5 \%)$ with pT3 and 38 of 62 patients $(61.3 \%)$ with pT4.

Finally, these data are in line with the results of Tarin and colleagues ${ }^{(21)}$ as they found that $\mathrm{LN}$ metastasis occurred in 18 of 312 patients $(6 \%)$ with <pT2, 16 of 88 patients $(18 \%)$ with pT2, 69 of 171 patients (40\%) with pT3, 12 of 20 patients $(60 \%)$ with pT4.

In our study, patients with higher stages pT3 or pT4 frequently had significant increased incidence of LN metastases above common iliac bifurcation.

These results are similar to Vazina and colleagues ${ }^{(12)}$ results as they had 64 patients with stage pT2, LN metastases were found exclusively in the pelvic region, except in two patients (3\%) with positive common iliac and aortic bifurcation LNs. Of patients with pT3 or pT4, 12 of 74 patients $(16 \%)$ had LN metastases outside the boundaries of a true pelvic lymph node dissection.

Dorin and colleagues ${ }^{(24)}$ showed that patients with higher stage $\geq \mathrm{T} 3 \mathrm{a}$ have more LNs metastasis above common iliac bifurcation (32 patients) (31\% of this group) than patients with organ confined disease $\leq \mathrm{T} 2 \mathrm{~b}$ (10 patients) (22\% of this group).

Finally Tarin and colleagues found that 6 of 312 patients with <pT2 disease $(2 \%), 5$ of 88 patients with pT2 disease (6\%), 28 of 171 patients with pT3 disease (16\%), and 3 of 20 patients with pT4 disease $(15 \%)$ were found to have $\mathrm{pN} 3$ disease. Overall, 42 patients $(7 \%)$ had $\mathrm{pN} 3$ disease.

In our study, we could not demonstrate any cases with skip lesion in the patients who had LNs metastasis above common iliac bifurcation.

Similar result was found by Abol-Enein and colleagues ${ }^{(23)}$ as they showed that 16 patients ( $8 \%$ of all patients) have LNs metastasis above common iliac bifurcation, all these patients have distal LNs metastasis at pelvis except one case $(0.5 \%$ of all patients) with single LN metastasis at common iliac LNs (skipped area) (they explained this due to sampling error).

Also Vazina and colleagues ${ }^{(12)}$ results showed that 14 patients ( $8 \%$ of all patients) have LNs metastasis above common iliac bifurcation; all these patients have distal LNs metastasis at pelvis except one case $(0.5 \%$ of all patients) (they explained this by direct drainage to the common iliac region).

Tarin and colleagues ${ }^{(21)}$ results showed that 42 patients ( $7 \%$ of all patients) had $\mathrm{pN} 3$ disease. Of these 42 patients, seven patients (1.1\% of all patients) had no positive lymph nodes within the true pelvis (skipped area). Since skip lesions are very rare, they explained this by missed positive LNs in the true pelvis or specimen-labeling error.

Dorin and colleagues ${ }^{(24)}$ results showed that 151 patients $(23 \%)$ had LN metastases at the time of cystectomy. Among LN-positive patients, 62 patients ( $9.5 \%$ of all patients) had positive LNs above the common iliac bifurcation. There were seven $\mathrm{LN}+$ patients ( $1 \%$ of all patients) who did not have any metastases below common iliac bifurcation (skipped area).

In contrast, Leissner and colleagues $^{(\mathbf{1 1})}$ identify 81 patients $(27.9 \%)$ with positive LNs. They found 20 of 290 patients $(6.9 \%$ of all patients), nodal metastases were located only at level 2 (between aortic bifurcation and common iliac bifurcation) without metastasis at distal level 1 (blew common iliac bifurcation). Positive LNs at only level 3 (above aortic bifurcation) were not encountered. So they strongly suggest that including these areas (common iliac and/or presacral regions) will optimize nodal staging.

Difference in the results of skipped lesion in different studies may be due to difference of number between studies, difference in surgical technique and due to the two limitations of mapping studies. First, it remains undetermined how many LNs are left behind and in which anatomic locations. Second, the area to which a removed $\mathrm{LN}$ is assigned may vary by surgeon. 
Regarding patients with only single LN metastasis, in our study, eight patients (12.3\%) had single positive lymph node and all positive lymph nodes were located below common iliac bifurcation. So we can use the term sentinel group to describe the following LNs groups' obturator, external iliac and internal iliac groups. These data are similar to Abol-Enein et al (23) results. They had involvement of a single lymph node in 22 cases $(45.8 \%$ of positive LN patients), of which all except 1 were within the endopelvic region (obturator, external iliac and internal iliac) (explained by sampling error) suggesting that there were no cases in which the primary drainage regions were skipped and disease landed in only secondary nodal sites. Thus, they defined the endopelvic site composed of the internal iliac, external iliac, and obturator groups of LNs as the sentinel regions.

In contrast, Dorin and colleagues showed that 57 patients with single LN metastasis and $93 \%$ of these patients had positive LNs below common iliac bifurcation only (level 1).

Leissner and colleagues ${ }^{(11)}$ found 29 patients having a single-node metastasis, and 10 of those 29 patients (34\%) had the metastasis outside the obturator and internal iliac lymph nodes.

We can explain this difference by low number of patients in our study and failure of mapping studies to determine the number of LNs that were left behind and in which location.

In our study, we found that positive lymph node affects significantly recurrence free survival and cancer specific survival.

This in agreement with Tarin et al (21) results as they showed that Patients with negative lymph nodes had significantly better Recurrence free survival and cancer specific survival than patients with positive lymph nodes $(\mathrm{p}<0.0005)$.

In our study, location of positive lymph nodes in different level of lymph nodes dissection did not significantly predict survival outcome as no significant difference between Patients with positive LN below common iliac bifurcation and patients with positive LN above common iliac bifurcation in CSS and RFS at 2 years ( $\mathrm{p}=0.795$ and $\mathrm{p}=$ 0.427 , respectively).

This is in agreement with Jensen and colleagues ${ }^{(\mathbf{2 5})}$ results as they showed that the presence of positive LNs above the bifurcation of the common iliac artery, without more cranial LN metastases, had no prognostic significance. Thus, anatomical localization of positive regional LNs had no prognostic value in their study.

Also Tarin and colleagues (21) showed that no evidence that RFS or CSS differed significantly between $\mathrm{pN} 1, \mathrm{pN} 2$, and $\mathrm{pN} 3$ and location of the positive node (ie, the common iliac region) did not provide additional prognostic information over the total number of positive lymph nodes.

Also steven and Poulsen ${ }^{(26)}$ showed that Survival was similar in this group of patients with lymphatic metastasis outside the boundaries of the standard pelvic lymph node dissection template compared to the entire population with lymph node metastasis.

In contrast, Dorin and colleagues showed that Patients with positive LNs above level 1 experienced significantly lower overall survival compared with patients with positive LNs confined to level 1 regions ( $\mathrm{p}=0.04)$, and a trend towards lower RFS ( $\mathrm{p}=0.12$ ). This difference may be attributed to low number of cases and short duration of follow up.

In our study, we found that difference in lymph node density in lymph node positive patients couldn't predict survival outcome as no significant difference was seen between patients with LN density $<20 \%$ and patients with LN density $>20 \%$ in CSS and RFS at 2 years $(\mathrm{p}=0.978$ and $\mathrm{p}=0.539$, respectively). This is in agreement with Jensen and colleagues (25) results as they showed that lymph node density had no prognostic factor but they use the threshold of $10 \%$. Also, Tarin and colleagues showed that Lymph node density was not a significant predictor of recurrence. (21)

On the other hand, May and colleagues (27) showed that lymph node density in patients with LN-positive bladder cancer has 
prognostic value, when a threshold value of $20 \%$ stratifies the population into two distinct groups. Also stein and colleagues ${ }^{(28)}$ showed that lymph node density was found to be a independent and significant prognostic factor. Patients with a lymph node density greater than $20 \%$, demonstrated a $17 \%$ recurrencefree survival at 10 years, compared to only a $43 \%$ at 10-year recurrence- free survival when the lymph node density was $20 \%$ or less. This difference may be attributed to low number of cases and short duration of follow up.

In our study, we found that the difference in number of positive lymph nodes did not affect the survival outcome as no significant difference between group of patients with one positive lymph node and group with more than one positive lymph bodes in CSS and RFS at 2 years $(\mathrm{p}=0.344)$ $(\mathrm{p}=0.406)$ respectively.

On the contrast, Jensen and colleagues (25) results showed that the number of positive LNs was a significant adverse prognostic factor; a threshold level of one positive $\mathrm{LN}$ being the most while stratifying patients with $>1$ positive LN did not add prognostic value. Thus, patients with 2 positive LNs had the same poor RFS and DSS as patients with $\geq 3$ positive LNs. Also Tarin and colleagues (21) showed that the number of positive lymph nodes (one, or two or more) was significantly associated with cancer-specific death. This difference may be attributed to low number of cases and short duration of follow up.

In our study, mean operative time $324 \pm 54.6$ minute and mean operative blood loss $850 \pm 180.4$ c.c. We had four cases $(6 \%)$ of lymphocele formation were seen postoperative. All of them were asymptomatic and detected by follow up ultrasound and managed conservatively except one case $(1.5 \%)$. This case was managed by percutaneous drainage ultrasound guiding. Only one case $(1.5 \%)$ of DVT was seen. Only one case $(1.5 \%)$ of pulmonary embolism was seen. Lymphoedema was not seen in any case.

Brössner and colleagues (29) compare between standard and extended lymphadenectomy groups to determine if extended lymphadenectomy increase morbidity of radical cystectomy? And they showed that there were no lymphoceles on clinical exploration in both groups. The median (range) operative duration was 277 (205-300) min in standard group and 330 (225-410) min in extended group $(P<0.01)$; thus extended lymphadenectomy increased the duration by $63 \mathrm{~min}$. Standard and extended Groups received a median (range) of $1.15(0-8)$ and $0.8(0-4)$ blood units $(P=$ $0.37)$.

Abol-Enein and colleagues ${ }^{(23)}$ in their study with all patients have undergone extended lymphadenectomy showed postoperatively 2 patients $(1 \%)$ died of a fatal pulmonary embolism. A nonfatal complications occurred in 16 patients $(8 \%)$, including deep vein thrombosis in 3 patients $(1.5 \%)$ and prolonged lymphatic drainage in $10(5 \%)$.

There are four important limitations of our study. First, the low numbers of cases that were involved in this study. Second, we do not have control group to compare the outcomes. Third, multiple factors affect the accuracy of lymph node positivity as surgical and pathological experience Also, the remaining number of lymph nodes that was left behind us remains unknown. Fourth, the short duration of follow up as the median follow up is 26 month but in a large multicenter series, Bochner et al. ${ }^{(30)}$ reported that the recurrence median time to was one year and $86 \%$ of recurrence occur within 3 years.

\section{Conclusion}

As no skip metastasis was found to level II and level III, we consider Level I LN groups (below common iliac bifurcation) as a sentinel groups for lymph nodes metastasis of bladder cancer. LN metastases in regions outside the boundaries of standard LND are common (40 \% of positive lymph node patients had metastasis in common iliac lymph nodes and presacral lymph nodes). Patients with positive common iliac lymph node metastasis removed at the time of radical cystectomy have a similar outcome when compared with patients with nodal disease limited to the true pelvis. Extended lymphadenectomy did not have significant 
morbidity. So we recommend inclusion of common iliac and presacral lymph nodes in routine lymphadenectomy during radical cystectomy to remove all metastatic tumor deposits completely and for better staging.

We found that patients with negative LN patients had better survival outcome than patients with positive LN but we did not find that the location of positive lymph nodes or lymph node density or number of positive lymph nodes significantly predicted outcome.

\section{References}

1. Bruins HM, Veskimae E, Hernandez V, et al. The Impact of the Extent of Lymphadenectomy on Oncologic Outcomes in Patients Undergoing Radical Cystectomy for Bladder Cancer: A Systematic Review. Eur Urol 2014; 66:1065-1077.

2. Leadbetter WF, Cooper JF. Regional gland dissection for carcinoma of the bladder; a technique for one-stage cystectomy, gland dissection, and bilateral uretero-enterostomy. J Urol 1950; 63: 242-60.

3. Godhard H, Koliopoulos A. La cystectomie totale chez la femme dans le cancer de la vessie. Rev Chir 1932;51:201-3.

4. Kerr WS, Colby FH. Pelvic lymph node dissection and total cystectomy in the treatment of carcinoma of the bladder. J Urol 1950;63:842-51.

5. Whitmore W, Marshall V. Radical total cystectomy for cancer of the bladder: 230 consecutive cases five years later. J Urol 1962;87:853-68.

6. Skinner DG (1982) Management of invasive bladder cancer: a meticulous pelvic node dissection can make a diVerence. J Urol 128:34-36.

7. Ghoneim MA, El-Mekresh MM, El-Baz MA, El-Attar IA and Ashamallah A: Radical cystectomy for carcinoma of the bladder: critical evaluation of the results in 1,026 cases. J Urol 1997; 158: 393.

8. Stein JP, Lieskovsky G, Cote R, et al. Radical cystectomy in the treatment of invasive bladder cancer: long-term results in 1,054 patients. J Clin Oncol 2001;19:666-75.

9. Dhar NB, Klein EA, Reuther AM, et al. Outcome after radical cystectomy with limited or extended pelvic lymph node dissection. J Urol 2008;179:873-8.

10. Smith JA, Whitmore Jr WF. Regional lymph node metastasis from bladder cancer. J Urol 1981;126:591-3.
11. Leissner J, Ghoneim MA, Abol-Enein H, et al. Extended radical lymphadenectomy in patients with urothelial bladder cancer: results of a prospective multicenter study. J Urol 2004; 171:139-44.

12. Vazina A, Dugi D, Shariat SF, Evans J, Link R, Lerner SP. Stage specific lymph node metastasis mapping in radical cystectomy specimens. J Urol 2004; 171:1830-4.

13. Roth B, Wissmeyer MP, Zehnder P, et al. A new multimodality technique accurately maps the primary lymphatic landing sites of the bladder. Eur Urol 2010;57:205-11.

14. Herr HW, Faulkner JR, Grossman HB, et al. Surgical factors influence bladder cancer outcomes: a cooperative group report. J Clin Oncol 2004;22:2781-9.

15. Konety BR, Joslyn SA, O'Donnel MA. Extent of pelvic lymphadenectomyand its impact on outcome in patients diagnosed with bladder cancer: analysis of data from the surveillance, epidemiology and end results program database. J Urol 2003;169:946-50.

16. Oken M. M., Creech R. H., Tormey D. C., et al. (2009): Toxicity and response criteria of the Eastern Cooperative Oncology Group. Am J Clin Oncol 5:649-655.

17. Sobin L.H., Gospodarowicz M.K., Wittekind C., et al. TNM classification of malignant tumors (UICC International Union Against Cancer). ed 7. New York, NY: WileyBlackwell; 2009: 262-265.

18. Shariat SF, Palapattu GS, Karakiewicz PI, et al. Discrepancy between clinical and pathologic stage: impact on prognosis after radical cystectomy. Eur Urol 2007; 51:13751, discussion 149-51.

19. Stenzl A, Cowan NC, De Santis M, et al. Treatment of muscle invasive and metastatic bladder cancer: update of the EAU guidelines. Eur Urol 2011; 59:1009-18.

20. Gakis G, Efstathiou J, Lerner SP, et al. ICUDEAU International Consultation on Bladder Cancer 2012: radical cystectomy and bladder preservation for muscle-invasive urothelial carcinoma of the bladder. Eur Urol 2013; 63:45-57.

21. Tarin TV, Power NE, Ehdaie B, et al. Lymph node-positive bladder cancer treated with radical cystectomy and lymphadenectomy: effect of the level of node positivity. Eur Urol 2012;61:1025-30.

22. Dangle P P., Gong M C, Bahnson R R. and Pohar K S. How Do Commonly Performed Lymphadenectomy Templates Influence 
Bladder Cancer Nodal Stage? J Urol 2010;183: 499-504.

23. Abol-Enein H, El-Baz M, Abd El-Hameed $\mathrm{MA}$, et al. Lymph node involvement in patients with bladder cancer treated with radical cystectomy: a pathoanatomical study - a single center experience. J Urol 2004;172:1818-21.

24. Dorin RP, Daneshmand S, Eisenberg MS, et al. Lymph node dissection technique is more important than lymph node count in identifying nodal metastases in radical cystectomy patients: a comparative mapping study. Eur Urol 2011; 60:946-52.

25. Jensen JB, Ulhoi BP, Jensen KM. Evaluation of different lymph node (LN) variables as prognostic markers in patients undergoing radical cystectomy and extended LN dissection to the level of the inferior mesenteric artery. BJU Int 2012; 109:38893.

26. Steven $\mathrm{K}$ and Poulsen AL. Radical Cystectomy and Extended Pelvic Lymphadenectomy: Survival of Patients With Lymph Node Metastasis Above the
Bifurcation of the Common Iliac Vessels Treated With Surgery Only. J Urol 2007; 178:1218-1224.

27. May M, Herrmann E, Bolenz, et al. Lymph Node Density Affects Cancer-Specific Survival in Patients with Lymph NodePositive Urothelial Bladder Cancer Following Radical Cystectomy. Eur Urol 2011; 59:712-719.

28. Stein JP, Cai J, Groshen S, Skinner DG: Risk factors for patients with pelvic lymph node metastases following radical cystectomy with en bloc pelvic lymphadenectomy: the concept of lymph node density. J Urol 2003; 170: 3541.

29. Brössner C, Pycha A, Toth A et al. Does extended lymphadenectomy increase the morbidity of radical cystectomy?.BJU 2004; 93:64-66.

30. Bochner BH, Kattan MW, Vora KC. Postoperative nomogram predicting risk of recurrence after radical cystectomy for bladder cancer. J Clin Oncol 2006;24:396772 . 